\title{
Efecto del tratamiento térmico en la morfología y conductividad térmica de cenizas volantes de la combustión de corbón
}

\author{
The Heat Treatment's Effect on the Morphology and the Fluing \\ Ash Thermal Conductivity from the Coal Combustion
}

\section{Efeito do tratamento térmico na morfologia e condutividade térmica de cinzas volantes da combustão de carvão}

\section{Resumen}

Se presenta el efecto del tratamiento térmico en la conductividad térmica efectiva $(\boldsymbol{k})$ a temperatura ambiente de polvos de cenizas volantes provenientes de la combustión de carbón de la Central Termoeléctrica de Termotasajero S.A. Dicho parámetro termofísico fue medido usando el sistema KD2Pro® Thermal Properties Analyzer, el cual funciona con el principio físico de flujo lineal transitorio de calor. El tratamiento térmico de las muestras se realizó usando una mufla eléctrica Ney® Vulcan D-130 para el rango de temperaturas de 1050, 1100, 1130, 1200, 1250 y $1300{ }^{\circ} \mathrm{C}$. La porosidad y densidad aparente fue hallada usando la norma NTC 4321-3. La morfología superficial de las muestras fue analizada usando Microscopía Electrónica de Barrido (MEB). Los resultados muestran que a medida que se incrementó la temperatura en el tratamiento térmico, las muestras cambiaron de color de gris oscuro a beige; disminuyó el porcentaje de porosidad, debido a su proceso de vitrificación, y se aumentó la densidad y, por ende, la conductividad térmica efectiva. Los valores reportados de $\boldsymbol{k}_{\boldsymbol{e}}$ son muy importantes en los cálculos del coeficiente de transmitancia térmica en los procesos de transferencia de calor donde se utilicen estas cenizas.

Palabras clave: Cenizas volantes, Conductividad térmica efectiva, Transmitancia térmica.

* Ph.D. Universidad Francisco de Paula Santander (San José de Cúcuta-Norte de Santander, Colombia). gpenaro@ufps.edu.co

** M.Sc. Universidad Francisco de Paula Santander (San José de Cúcuta-Norte de Santander, Colombia). maryaferr@gmail.com

*** Ph.D. Universidad Francisco de Paula Santander (San José de Cúcuta-Norte de Santander, Colombia). hjdulce@ufps.edu.co 


\section{Abstract}

This paper presents the heat treatment's effect, on the effective thermal conductivity $\left(\mathbf{k}_{\mathbf{e}}\right)$ at room temperature, of the flying dust ashes, from the Termotasajero S.A's coal combustion thermoelectric. The above mentioned thermo-physical parameter, was measured using the Thermal Properties Analyzer KD2Pro® system, which works with the linear transitory heat flow's physical principle. The samples' heat treatment, were performed using an electrical muffle Ney® Vulcan D-130 for the 1050, 1100, 1130, 1200, 1250 and $1300^{\circ} \mathrm{C}^{\prime}$ s temperature ranges. The porosity and the apparent density were found using the standard NTC 4321-3.

The samples' superficial morphology was analyzed using a scanning electronic microscopy (SEM). Our results show that as the temperature increased in the heat treatment, the samples changed color from a dark brown to a beige gray. It also decreased the porosity percentage, due to the vitrification process, increasing the density too, and consequently the thermal conductivity effectiveness. The $\mathrm{k}_{\mathrm{e}}$ values found are very important for the thermal transmittance calculations' coefficient (U-value), in the heat transfer processes, where these ashes are used.

Keywords: Effective Thermal Conductivity, Fly Ash, Thermal transmittance

\section{Resumo}

Apresenta-se o efeito do tratamento térmico na condutividade térmica efetiva (ke) a temperatura ambiente de poeiras de cinzas volantes provenientes da combustão de carvão da Central Termoeléctrica de Termotasajero S.A. Este parâmetro termofísico foi medido usando o sistema KD2Pro ${ }^{\circledR}$ Thermal Properties Analyzer, o qual funciona com o princípio físico de fluxo lineal transitório de calor. O tratamento térmico das amostras se realizou usando uma mufla elétrica Ney® Vulcan D-130 para a faixa de temperaturas de 1050, 1100, 1130, 1200, 1250 e 1300 ${ }^{\circ} \mathrm{C}$. A porosidade e densidade aparente foram achadas usando a norma NTC 4321-3. A morfologia superficial das amostras foi analisada usando Microscopia Eletrônica de Varredura (MEV). Os resultados mostram que na medida em que se incrementou a temperatura no tratamento térmico, as amostras mudaram de cor de cinza escura a bege; diminuiu a porcentagem de porosidade, devido a seu processo de vitrificação, e se aumentou a densidade e, portanto, a condutividade térmica efetiva. Os valores reportados de ke são muito importantes nos cálculos do coeficiente de transmitância térmica nos processos de transferência de calor onde se utilizam estas cinzas.

Palavras chave: Cinzas volantes, Condutividade térmica efetiva, Transmitância térmica. 


\section{INTRODUCCIÓN}

La Central Termoeléctrica de Termotasajero S.A, ubicada en el área metropolitana de San José de Cúcuta (Norte de Santander, Colombia), produce gran cantidad de cenizas volantes producto de la combustión de carbón. En la actualidad, dichas cenizas son utilizadas principalmente en la industria cementera como sustituto del clinker en la fabricación de cemento Portland, lo cual reduce costos y disminuye la contaminación por $\mathrm{CO}_{2}$ asociada a la producción de cemento $[1,2]$. Otras aplicaciones de las cenizas volantes están en la remoción del ácido bórico y de sales de boro del agua usada por las industrias de vidrios, de porcelanas, de producción de cueros y de cosméticos $[3,4]$.

El conocimiento de la conductividad térmica efectiva $\left(\boldsymbol{k}_{e}\right)$ de un material, la cual indica la cantidad de calor (energía térmica) que pasa entre dos puntos dentro de este por unidad de área, tiempo y gradiente de temperatura [5-7], es de vital importancia para el cálculo de los coeficientes de impedancia térmica en los ambientes donde se utilice dicho material [8]. Para el caso de muestras en polvo, dicho parámetro depende de la distribución de tamaño de partícula, morfología, estructura y composición química [9].

En este trabajo se estudia el comportamiento de la conductividad térmica efectiva a temperatura ambiente, y la morfología de muestras prensadas de cenizas volantes de Termotasajero S.A., sometidas a cocción en un rango de temperatura entre $1050{ }^{\circ} \mathrm{C}$ y $1300{ }^{\circ} \mathrm{C}$.

\section{Materiales Y MÉTOdos}

Con los polvos de cenizas volantes suministrados por la Central Termoeléctrica de Termotasajero se elaboraron muestras cilíndricas prensadas uniaxialmente a 100 Psi, de $12 \mathrm{~cm}$ de altura y $3,6 \mathrm{~cm}$ de diámetro, las cuales fueron humectadas con agua en una concentración del $58 \%$ en peso. Una vez compactadas las muestras, se secaron a temperatura ambiente durante 24 horas; luego, se introdujeron en una estufa de circulación forzada a $50^{\circ} \mathrm{C}$ por un tiempo similar y posteriormente a $105^{\circ} \mathrm{C}$ durante otras 24 horas, período en el cual la masa se mantuvo constante. El proceso de cocción se realizó usando una mufla eléctrica marca Ney ${ }^{\circledR}$ Vulcan
D-130 para las temperaturas: 1050, 1100, 1130, 1200, 1250 y $1300{ }^{\circ} \mathrm{C}$, durante una hora. En la Figura 1 se presenta la imagen digital de las probetas, tanto para la muestra seca como para la muestra tratada a $1300{ }^{\circ} \mathrm{C}$.

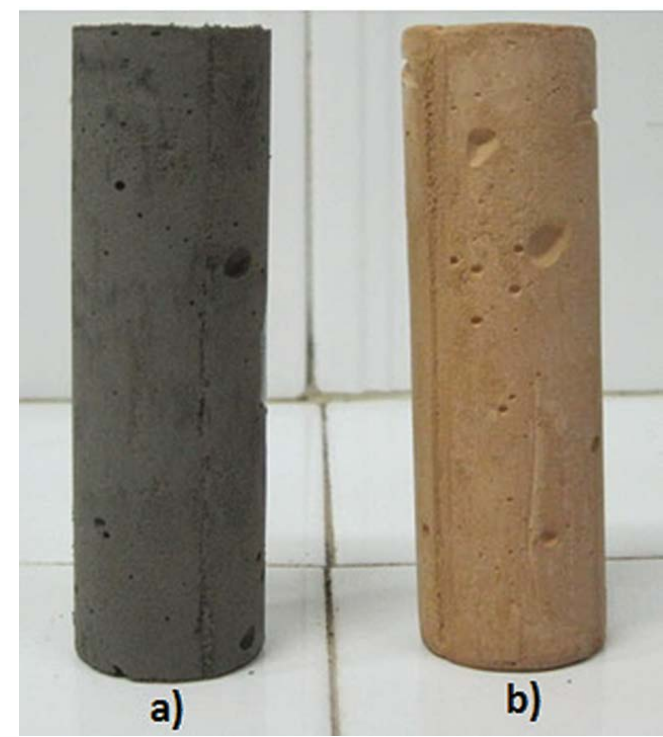

Fig. 1. Imagen digital de probeta compactada de ceniza volante, a) sin tratamiento térmico, b) sinterizada a $1300^{\circ} \mathrm{C}$.

En total se elaboraron cinco probetas para cada temperatura, a las cuales se les determinó la densidad aparente usando la relación masa sobre volumen y la porosidad o porcentaje de absorción de agua según la norma NTC 4321-3, la cual consiste en medir la masa de la muestra seca, luego introducirla durante una hora en agua a $100{ }^{\circ} \mathrm{C}$, posteriormente sacarla y sumergirla durante otra hora en agua a temperatura ambiente, luego extraerla, secarla con un paño de papel y medirle la masa húmeda; con los datos anteriores se determina el porcentaje de absorción de agua, que es considerado como la porosidad presente en la muestra.

La morfología de las muestras fue estudiada usando un microscopio electrónico de barrido FEI Quanta. Para determinar la conductividad térmica efectiva de las muestras se usó el sistema $\mathrm{KD} 2 \mathrm{Pro} \AA$, que cumple con la norma ASTM D5334-08; dicho sistema funciona con el principio físico de flujo lineal transitorio de calor, que consiste en introducir una fuente lineal de calor en la muestra, y medir, en el estado transitorio de temperaturas, las variaciones del gradiente de temperatura en el tiempo [10]. 


\section{RESULTADOS Y DISCUSIÓN}

En la Tabla 1 se reportan los promedios de la densidad aparente y del porcentaje de porosidad para las muestras de ceniza volante sometidas a las temperaturas de cocción antes descritas. Estos promedios señalan que la densidad aparente aumenta a medida que se incrementa la temperatura de cocción en la muestra; los resultados hallados concuerdan con lo reportado por V. Kumar [11], que establece que dicho parámetro para las cenizas volantes debe estar entre 0,9 y $1,3 \mathrm{~g} / \mathrm{cm}^{3}$. Por su parte, el porcentaje de porosidad presente en la muestra disminuye al incrementarse la temperatura del tratamiento térmico en la muestra, lo anterior se debe a la disminución de la porosidad por la vitrificación y sinterización de las partículas de ceniza volantes (cenosferas y plerosferas), que se observa en el análisis de la morfología superficial de las muestras usando MEB.

\section{TABLA 1}

DENSIDAD APARENTE Y PORCENTAJE DE POROSIDAD PARA CADA TRATAMIENTO TÉRMICO

\begin{tabular}{ccc}
\hline $\begin{array}{c}\text { Temperatura de } \\
\text { cocción }\left({ }^{\circ} \mathbf{C}\right)\end{array}$ & Densidad aparente $\left(\mathbf{g} / \mathbf{c m}^{3}\right)$ & $\begin{array}{c}\text { Porosidad } \\
(\mathbf{\%})\end{array}$ \\
\hline $\mathbf{1 0 5 0}$ & $1,145 \pm 0,015$ & $44,516 \pm 0,029$ \\
$\mathbf{1 1 0 0}$ & $1,172 \pm 0,014$ & $39,348 \pm 0,028$ \\
$\mathbf{1 1 3 0}$ & $1,192 \pm 0,014$ & $38,701 \pm 0,028$ \\
$\mathbf{1 2 0 0}$ & $1,199 \pm 0,014$ & $37,644 \pm 0,028$ \\
$\mathbf{1 2 5 0}$ & $1,224 \pm 0,015$ & $36,638 \pm 0,027$ \\
$\mathbf{1 3 0 0}$ & $1,226 \pm 0,015$ & $34,862 \pm 0,027$ \\
\hline
\end{tabular}

Los resultados de la conductividad térmica efectiva $\left(\boldsymbol{k}_{e}\right)$ a temperatura ambiente de las muestras sometidas a las diferentes temperaturas de cocción se presentan en la Tabla 2; se aprecia un comportamiento creciente de dicho parámetro termofísico al aumentar la temperatura del tratamiento térmico, lo cual es consistente con los resultados hallados para la densidad, pues al incrementarse ésta es de esperarse un incremento en $\boldsymbol{k}_{e}[11]$.

\section{TABla 2}

CONDUCTIVIDAD TÉRMICA EFECTIVA A TEMPERATURA AMBIENTE PARA LAS PROBETAS SOMETIDAS A TRATAMIENTO TÉRMICO EN EL RANGO QUE VA DE $1050{ }^{\circ} \mathrm{C}$ A $1300{ }^{\circ} \mathrm{C}$

\begin{tabular}{cc}
\hline $\begin{array}{c}\text { Temperatura de cocción } \\
\left({ }^{\circ} \boldsymbol{C}\right)\end{array}$ & $\begin{array}{c}\text { Conductividad térmica efectiva } \\
\boldsymbol{K}\left(\boldsymbol{W} / \boldsymbol{m}^{\circ} \boldsymbol{C}\right)\end{array}$ \\
\hline $\mathbf{1 0 5 0}$ & $0,082 \pm 0,004$ \\
$\mathbf{1 1 0 0}$ & $0,096 \pm 0,003$ \\
$\mathbf{1 1 3 0}$ & $0,098 \pm 0,003$ \\
$\mathbf{1 2 0 0}$ & $0,111 \pm 0,002$ \\
$\mathbf{1 2 5 0}$ & $0,113 \pm 0,003$ \\
$\mathbf{1 3 0 0}$ & $0,118 \pm 0,004$ \\
\hline
\end{tabular}

El comportamiento de $\boldsymbol{k}_{e}$ en función de la densidad y la porosidad para las muestras estudiadas se presenta en las Figuras 2 y 3, respectivamente; de estas se infiere que a medida que la densidad se incrementa, $\boldsymbol{k}_{\boldsymbol{e}}$ aumenta; mientras que a mayor porosidad menor es el valor para $\boldsymbol{k}_{\boldsymbol{e}}$, lo cual es consistente con lo reportado en la literatura, pues a mayor cantidad de aire presente en la muestra menor debe ser el valor de $\boldsymbol{k}_{\boldsymbol{e}}$, ya que el aire actúa como una barrera al flujo del calor [6]. 


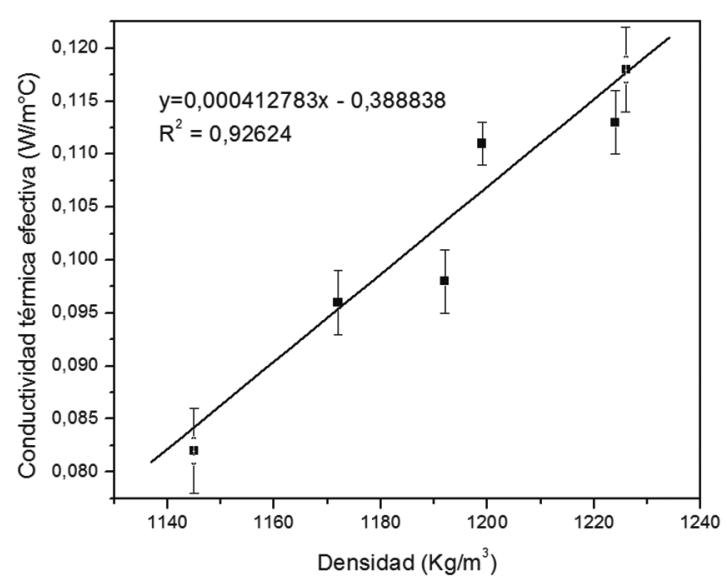

Fig. 2. Conductividad térmica efectiva de las cenizas volantes en función de la densidad.

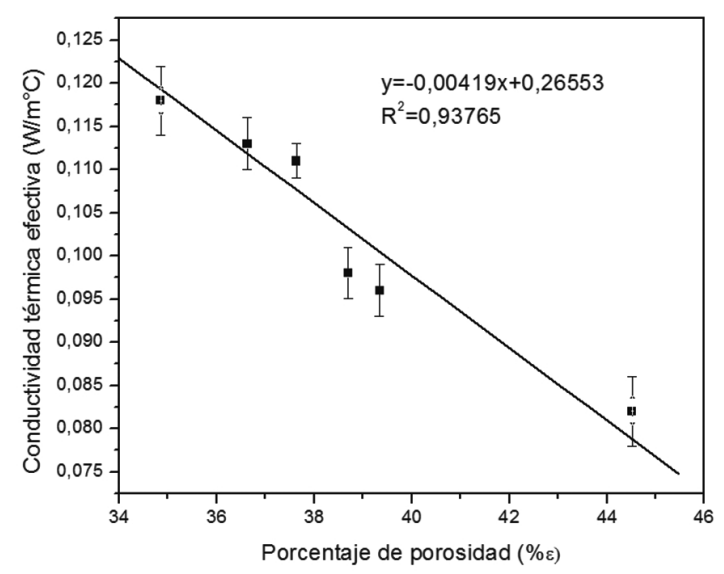

Fig. 3. Conductividad térmica efectiva de las cenizas volantes en función del porcentaje de porosidad.
Un análisis del aspecto superficial de las probetas en función de la temperatura del tratamiento térmico se presenta en la Figura 4, en la que se aprecia cómo a medida que se incrementa la temperatura de cocción las muestras pasan de color gris oscuro (sin tratamiento térmico) a beige claro; este efecto también puede observarse en la Figura 1. Lo anterior se debe a la eliminación de los inquemados y del materia volátil presentes en la muestra, así como a los óxido de hierro (hematita) presentes en la ceniza volante [12], lo cual se evidencia en el espectro de dispersión de energías de rayos X, presentado en la Figura 5, para la muestra sin tratamiento térmico, y a la atmósfera oxidante presente en el proceso de sinterización.

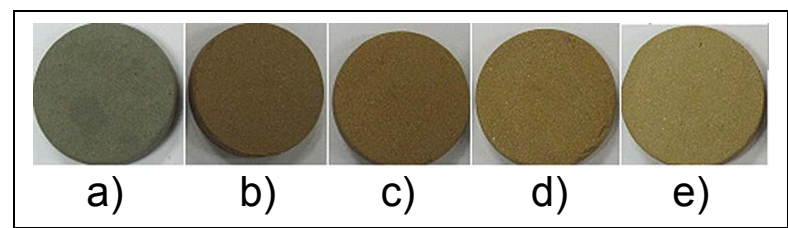

Fig. 4. Imagen digital de un corte transversal a las muestras, a) sin tratamiento térmico, b) $1050{ }^{\circ} \mathrm{C}, \mathrm{c}$ ) $\left.\left.1100{ }^{\circ} \mathrm{C}, \mathrm{d}\right) 1200^{\circ} \mathrm{C} \mathrm{y} \mathrm{d}\right) 1300{ }^{\circ} \mathrm{C}$.

El análisis morfológico de la superficie de las muestras, usando Microscopía Electrónica de Barrido (MEB), se presenta en la Figura 5. 


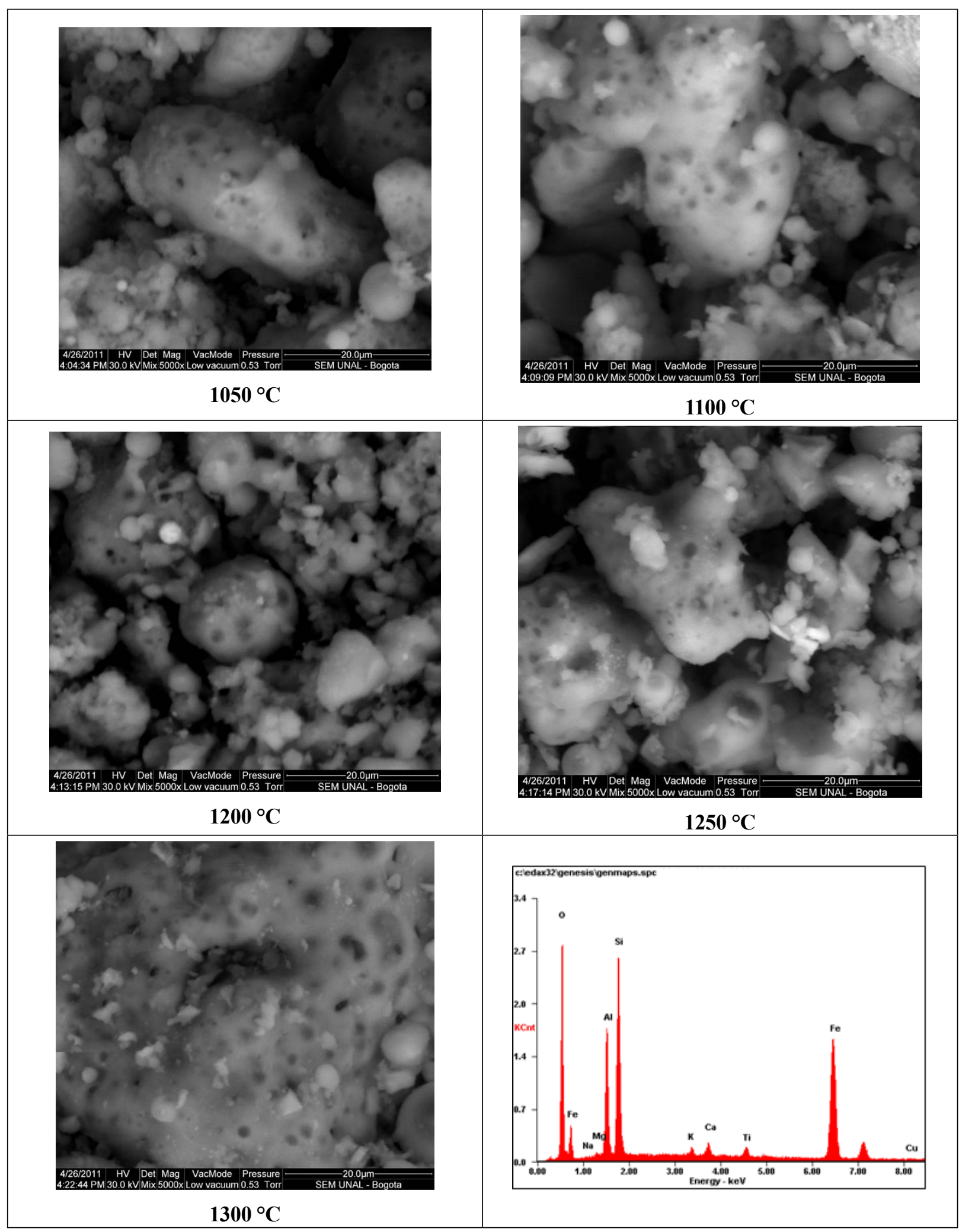

Fig. 5. Microfotografías a 5000X, usando MEB FEI Quanta, para las muestras sometidas a temperaturas de $1050{ }^{\circ} \mathrm{C}$, $1100^{\circ} \mathrm{C}, 1200^{\circ} \mathrm{C}, 1250^{\circ} \mathrm{C}$ y $1300^{\circ} \mathrm{C}$, y espectro de dispersión de rayos x (EDX) para la muestra sin tratamiento térmico. 
En la Figura 5 se observan microfotografías a 5000X tomadas con el sensor de electrones secundarios del MEB a $30 \mathrm{kV}$, para las muestras sometidas a tratamientos térmicos de $1050{ }^{\circ} \mathrm{C}, 1100{ }^{\circ} \mathrm{C}, 1200{ }^{\circ} \mathrm{C}$, $1250^{\circ} \mathrm{C}$ y $1300^{\circ} \mathrm{C}$. Se aprecia en la figura que a medida que la temperatura del tratamiento térmico aumenta, la sinterización o vitrificación de las partículas constituyentes de la ceniza volante se incrementa; lo anterior se debe al proceso de vitrificación de la muestra, en especial de la mullita y el cuarzo [13], proceso que se evidencia en la microfotografía sinterizada a $1300{ }^{\circ} \mathrm{C}$, donde se aprecia el aspecto vidrioso de la superficie de la muestra. Dicho proceso de vitrificación es consistente con los resultados hallados para la porosidad, pues a medida que se incrementó la temperatura de cocción, la porosidad disminuyó, incrementándose la contracción de la muestra. Por otra parte, dicho comportamiento también permite explicar el comportamiento de $\boldsymbol{k}_{\boldsymbol{e}}$, pues al existir menor porosidad, existirá menor cantidad de aire en el interior de la muestra, permitiendo que la conducción de calor a través de ésta se incremente.

\section{Conclusiones}

A medida que la temperatura del tratamiento térmico se incrementa, la porosidad presente en las muestras disminuye, y aumenta su densidad y, por ende, la conductividad térmica efectiva $\left(\boldsymbol{k}_{e}\right)$; encontrándose que los valores reportados para este parámetro térmico están en el rango reportado en la literatura. Por otra parte, se evidenció el proceso de vitrificación de las muestras de ceniza volante al incrementarse la temperatura del tratamiento térmico. Los valores de $\boldsymbol{k}_{\boldsymbol{e}}$ hallados en este trabajo para las muestras de ceniza volante son muy importantes para determinar los coeficientes de transmitancia térmica (U-value) de los elementos constructivos que se elaboren usando dichas cenizas volantes (por ejemplo, ladrillos refractarios), parámetro utilizado en el cálculo de las pérdidas energéticas en los procesos de transferencia de calor.

\section{Agradecimientos}

Al Fondo de Investigaciones Universitarias FINUUFPS, por su apoyo económico. Al Ingeniero Yesid Peña, por su colaboración en la preparación de las muestras.

\section{REFERENCIAS}

[1] A. Bilodeau and V. Mohan-Malhotra. "HighVolume Fly Ash System: Concrete Solution for Sustainable development". Materials Journal, Vol. 97 (1): 41-48, 2000.

[2] J. Davidovits "Global Warming Impact on the Cement and Aggregates Industries". World Resource Review. Vol. 6(2): 263-278, 1994.

[3] N. Öztürk and D. Kavak. "Adsorption of boron from aqueous solutions using fly ash: Batch and column studies". Journal of Hazardous Materials. B127. 81-88, 2005.

[4] H. Polat, A. Vengosh, I. Pankratov and M. Polat. "A new methodology for removal of boron from water by coal and fly ash". Desalination. Vol. 164, 173-178, 2004.

[5] Y. S. Touloukian, R. W. Powell, C. Y. Ho and P. G. Klemens. "Thermal Conductivity, Thermophysical properties of matter". Volume 2. IFI/PLENUM. New York. 3p, 1970.

[6] T.M.I. Mahlia, B.N. Taufiq, Ismail, H.H. Masjuki. "Correlation between thermal conductivity and the thickness of selected insulation materials for building wall". Energy and Buildings. Volume 39, Issue 2, 182-187, 2007.

[7] J.K. Carson, S.J. Lovatt, D.J. Tanner and A.C. Cleland. "Thermal conductivity bounds for isotropic porous materials". International Journal of Heat and Mass Transfer. Vol. 48, 2150-2158, 2005.

[8] B. Anderson. En ISO 6946:1997, "Building components and building elements -thermal resistance and thermal transmittanceCalculation methods", 2006.

[9] G. Peña Rodríguez et al. "Conductividad térmica efectiva promedio de polvos de arcillas utilizadas en la industria cerámica del área metropolitana de san José de Cúcuta”. Revista Colombiana de Física, Vol. 40, 278-280, 2008. 
[10] J.C. Jaeger and J.H. Sass. "A line source method for measuring the thermal conductivity and diffusivity of cylindrical specimens of rock and other poor conductors". British Journal of Applied Physics. Volume 15, Number 10, 1964.

[11] V. Kumar. Fly Ash Unit. Department of Science \& Techonology India. NRRDA Workshop. New Delhi, 2012.
[12] R. Demirboga and R. Gül. "The effects of expanded perlite aggregate, silica fume and fly ash on the thermal conductivity of lightweight concrete". Cement and Concrete Research. Vol. 33, 723-727, 2003.

[13] L.R. Ortega Triana. "Caracterización estructural y térmica de cenizas volantes de la combustión de carbón". Plan de estudios Ing. Minas. UFPS, 2014. 